erythematosus, polymyositis, spondyloarthritis, systemic sclerosis (ES) or rheumatoid arthritis (RA) has been reported.

Objectives: To describe the clinical characteristics of 15 patients with sarcoidosis and chronic inflammatory rheumatic diseases in 6 hospitals of Catalonia.

Methods: Retrospective descriptive study of patients affected by sarcoidosis and chronic inflammatory rheumatic diseases. The following data were recorded: sex, age, date of diagnosis and clinical characteristics, treatment and evolution of both pathologies. As well as the results of the pulmonary $\mathrm{CT}$, the angiotensin converting enzyme (ACE) and the biopsy results.

Results: We described 15 patients with sarcoidosis, 6 men and 9 women, median age 58 years (39-84). Five patients had seropositive RA (rheumatoid factor and citrullinated antipeptide antibodies), 2 ankylosing spondylitis, 2 psoriasic arthritis, 3 systemic sclerosis, 2 Sjögren's syndrome and 1 dermatomyositis and primary biliary cirrhosis. The diagnosis of sarcoidosis preceded that of rheumatic disease in 5 patients with a mean time of 11.2 years (2-27). It was found to be concomitant in 3 cases and later in 7 patients with an average time difference of 7 years (2-19). At the time of diagnosis of sarcoidosis, 3 patients received treatment with biologic drugs (etanercept, abatacept and golimumab). Fifteen patients presented adenopathies (14 mediastinal and 1 retroperitoneal), 8 lung involvement (micronodullillar interstitial pattern in 4 , pulmonary nodules in 2 and unspecified 2), 1 pleural effusion, 8 cutaneous manifestations (2 erythema nodosum, 5 cutaneous sarcoidosis and 1 scar sarcoidosis), 1 muscular pattern with muscle enzymes elevation, 2 lower limb arthritis, 1 meningitis, 1 hepatosplenomegaly, 1 panuveitis and 1 posterior uveitis. The ACE values were high in 8 cases. The histopathological diagnosis of sarcoidosis was made by lung biopsy in 6 patients, ganglionar in 5 , cutaneous in 3 and muscular in 1 . Ten patients received systemic glucocorticoid treatment with improvement of clinical manifestations and imaging tests. One patient who presented pulmonary involvement also received mycophenolate and another with joint involvement received methotrexate and leflunomide.

Conclusion: A diagnosis of sarcoidosis is often challenging. We must not forget to include it in the differential diagnosis of lung affectation of our patients with chronic inflammatory rheumatic diseases and in particular in those who receive treatment with biological drugs.

Disclosure of Interests: Marta Valls Roc: None declared, Meritxell Sallés Lizarzaburu: None declared, Sonia Mínguez Blasco: None declared, Elisabet Garcia Casares: None declared, Elena Riera Alonso: None declared, Vera Ortiz-Santamaria Speakers bureau: GSK, Roche, Pfizer, MSD, Clara Sanguesa: None declared, Alejandro Olive: None declared DOI: 10.1136/annrheumdis-2019-eular.2834

\section{AB1109 CHARACTERIZATION OF PATIENTS WITH INTERSTITIAL PNEUMONIA WITH AUTOIMMUNE FEATURES (IPAF) AND ITS COMPARISON WITH PATIENTS WITH SCLERODERMA-RELATED INTERSTITIAL LUNG DISEASE AND WITH IDIOPATHIC FIBROSIS}

Karen Vergara ${ }^{1}$, Silvana Saavedra ${ }^{1}$, Felipe Reyes ${ }^{2}$, Annelise Goecke ${ }^{1}$, Caterina Chesta ${ }^{3}$, Sebastian Chavez ${ }^{4}{ }^{1}$ Hospital Clinico Universidad de Chile, Reumatologia, Santiago, Chile; ${ }^{2}$ Hospital Clinico Universidad de Chile, Neumologia, Santiago, Chile; ${ }^{3}$ Instituto Nacional del Cancer, Medicina Interna, Santiago, Chile; ${ }^{4}$ Hospital Clinico Universidad de Chile, Medicina Interna, Santiago, Chile

Background: Diffuse parenchymal pulmonary diseases, called interstitial lung diseases, are a heterogeneous group of disorders that are classified together due to clinical, radiographic, physiological or similar pathological manifestations ${ }^{1}$.The diagnosis of idiopathic interstitial pneumonias requires the exclusion of known causes of interstitial pneumonia. Identifying an underlying etiology is important for clinical perspectives because it impacts prognosis and treatment ${ }^{2}$. A recent number of studies has shown that many patients diagnosed as idiopathic interstitial pneumonia have clinical elements that suggest an underlying autoimmune process without meeting established diagnostic criteria for connective tissue disease ${ }^{3,4}$

Objectives: Our objectives were characterize the clinical findings of patients who meet the IPAF criteria and compare them with the clinical characteristics of patients with scleroderma-related interstitial lung disease and patients with idiopathic pulmonary fibrosis.
Methods: We retrospectively reviewed 254 patients hospitalized at the Hospital Clínico de La Universidad de Chile between January 2012 and June 2018 who had ICD-10 diagnosis of J.84 (Other respiratory diseases principally affecting the interstitium) and J99.1 (Respiratory disorders in other diffuse connective tissue disorders). The electronic medical record was reviewed retrospectively to extract pertinent data. We applied IPAF criteria to this 254 patients. We then characterized the clinical, serological and morphological features of the IPAF cohort and compared outcomes to other ILD cohorts: scleroderma-related interstitial lung disease and idiopathic pulmonary fibrosis (IPF)

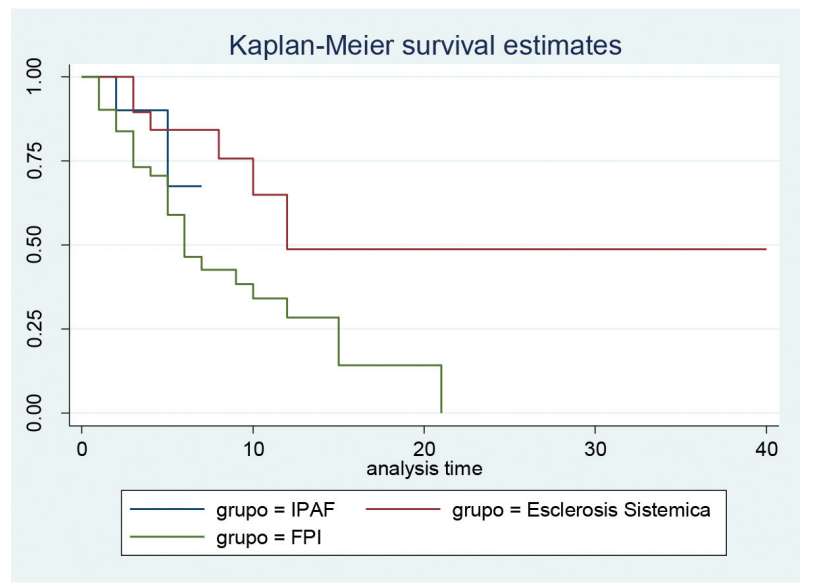

Figure 1

Results: Of 254 patients screened, 17 patients met the IPAF criteria. Mean age was 60 years with a female predominance. The most frequent pattern by high-resolution computed tomography was NSIP present in $46.7 \%$. The median of Forced Vital Capacity was $82 \%$, and median of DLCO was $50 \% .14$ patients (82\%) were treated with corticosteroids. 11 Patients (64\%) used other immunosuppressant: 6 patients azathioprine, 4 mycophenolate and 1 patient used cyclophosphamide. One patient received a lung transplant in IPAF cohort. We identified 2 deaths in IPAF cohort, 6 in sclerosis systemic and 30 in IPF cohort. IPAF cohort survival was worse than Scleroderma cohort and better than the IPF cohort.

Conclusion: Our IPAF cohort is similar to the cohorts described in other studies, in relation to the age of diagnostic, female predominance and High-resolution Computed Tomograph pattern. Also the trend in survival was similar to others previously described. Our study have limitations, the first one is related to the retrospective nature of the reviewed cohorts. Further prospective studies should be conducted for a more comprehensive evaluation of the evolution of these diseases and the impact of the treatments used.

\section{REFERENCES}

[1] Travis, W. (2002). American Thoracic Society/European Respiratory Society Multidisciplinary Consensus Classification of the Idiopathic Interstitial Pneumonias. Am J Respir Crit Care Med, 165, 277-304.

[2] Park, J. (2007). Prognosis of fibrotic interstitial pneumonia: idiopathic versus collagen vascular disease-related subtypes. Am J Respir Crit Care Med; 175: 705-711.

[3] Corte, T. 2012. Significance of connective tissue disease features in idiopathic interstitial pneumonia. Eur Respir J 2012; 39: 661-668.

[4] Vij, R. (2011). Autoimmune-featured interstitial lung disease: A distinct entity. Chest; 140:1292-1299.

Disclosure of Interests: Karen Vergara: None declared, Silvana Saavedra: None declared, Felipe Reyes: None declared, Annelise Goecke Consultant for: Roche, abbvie, novartis, Phizer, Paid instructor for: Roche, Speakers bureau: Roche, Novartis, Abbvie, pfizer, Caterina Chesta: None declared, Sebastian Chavez: None declared

DOI: 10.1136/annrheumdis-2019-eular.7794 